\title{
Reduction of multi-photon ionization in dielectrics due to collisions
}

\author{
D. Du, X. Liu, and G. Mourou \\ Center for Ultrafast Optical Science, The University of Michigan, 2200 Bonisteel Blvd., Room 1006, Ann Arbor, MI 48109-2099, USA \\ TEL: + 001-313 763-4875, FAX: + 001-313 763-4876
}

Received: 20 September 1995/accepted: 25 April 1996

\begin{abstract}
The collisional effect due to the multi-photon ionization process in dielectric material has been studied. We found that the breakdown threshold of fused silica is the same for both linearly and circularly polarized light at $55 \mathrm{fs}$ and $100 \mathrm{fs}$, which we believe is an indication of the suppression of multi-photon ionization in solids. By numerically solving the time-dependent Schrödinger equation with scattering, for the first time, we have observed substantial reduction of the multi-photon ionization rate in dielectrics due to collisions.
\end{abstract}

PACS: $32.80 . \mathrm{Rm} ;$ 77.32.Jp

Multi-photon ionization (MPI) of free atoms is photoionization with photon energies smaller than the ionization potential. MPI has been uninterruptedly studied both experimentally and theoretically since its discovery in $1965[1,2]$. Experimentally, important results on ionization rate as a function of laser intensity were obtained in the 1970s [3]. The observation of ejected electrons with excessive photon energy than the minimum MPI required (termed as above-threshold ionization) widened the understanding of laser-atom interaction [4-6]. Multiphoton Ionization of Atoms, edited by Chin and Lambropoulos and Atoms in Intense Laser Fields, edited by Gavrila have covered a great deal of the pursuit. In the theoretical studies of MPI, both analytical approximation and numerical methods have been employed. Keldysh studied the ionization probability of a hydrogen atom in an electromagnetic field, where the final state of the electron is described by the so-called Volkov states [7]. Depending on the adiabatic parameter, $\gamma=\left(U_{\mathrm{i}} / 2 U_{\mathrm{p}}\right)^{1 / 2}$, where $U_{\mathrm{i}}$ is the ionization potential and $U_{\mathrm{p}}=e^{2} E^{2} / 4 m \omega^{2}$ is the quiver energy of an electron in the electric field, the ionization is related to the tunneling effect for $\gamma \ll 1$, or to multiphoton absorption when $\gamma \gg 1$. Keldysh's work was rediscovered and improved by Faisal and Reiss later and they are now known as the KFR theory $[8,9]$. Numerical studies of the time-dependent Schrödinger equation have also been done $[10,11]$. By numerically integrating the time-dependent Schrödinger equation, one can directly calculate the ionization of an atom in the electronmagnetic field. In both approaches, researchers were able to explain many of the observations of multi-photon processes.

We would like to point out, however, that most of the experiments on MPI were done with low pressure gases where inter-atomic distance is larger than the electron excursion amplitude, hence collisional effect is negligible, and theoretical studies were also concentrated on single atoms interacting with the laser field.

Recently, we performed a series of laser-induced breakdown (LIB) experiments with pulse widths ranging from 150 fs to $7 \mathrm{~ns}$ in wide-hand-gap dielectric materials [12]. The normally transparent dielectric material can be ionized by the intense laser radiation and absorbs the photon energy, resulting in a catastrophic breakdown of the material. The key factor in LIB is the free electron generation. Since the photon energy is much smaller than the energy gap of the material, the electron generation is through nonlinear processes. The main mechanisms for free electron generation are avalanche ionization and multi-photon ionization. Our findings show that avalanche ionization dominates the breakdown, and multiphoton ionization effect is small even at the short pulse width regime where one would expect that MPI is much stronger due to the high intensity of the laser field. It puzzles us and motivates us to further investigate the photoinization process in solids. In solids and dense gases, the excursion amplitude of a valence electron can become bigger than the inter-atomic distance when high intensity laser pulses are applied. Therefore, scattering of the electron off the neighboring atom is likely to occur during an optical cycle. Because of the high collision frequency inside solids, the periodic motion of electrons has been disturbed, and the electrons are dephased with respect to the driven field. Hence we believe that MPI will be suppressed in solids.

In this paper we report our investigation on MPI in solids through laser-induced breakdown experiments and by numerical integration of the time-dependent Schrödinger equation. We performed laser-induced breakdown 
experiments with both linearly and circular polarized light. A chirped pulse amplification laser system was used in the experiment [13]. The laser pulse widths were $55 \mathrm{fs}$ and $100 \mathrm{fs}$ full width at half maximum (FWHM). Fused silica samples were made from Corning 7940 , with a thickness of $150 \mu \mathrm{m}$. The detailed description of the experimental setup can be found elsewhere [12]. Two diagnostics were used to identify the onset of the breakdown. We observed the decrease of the transmission due to reflection from the formed critical density plasma as well as the emission from the plasma at the focus spot. The results are shown in Fig. 1. The breakdown threshold decreases as pulse width decreases from the nanosecond regime to

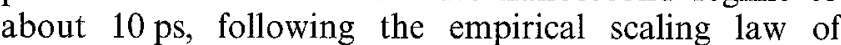
$F_{\text {th }} \propto \sqrt{\tau}$. However, as pulse width becomes shorter than a picosecond, the threshold did not decrease, which indicates that multi-photon ionization is not a dominant process. The solid line in Fig. 1 is based on avalanche ionization theory in the DC limit by Thornber [14] and assuming that the initial electron density is on the order of $10^{8} / \mathrm{cm}^{3}$. The deviation from the DC avalanche ionization theory is due to the multi-photon ionization contribution to the initial electron generation. Multi-photon ionization takes place at the very leading edge of the pulse. When the laser intensity becomes so high that the electron excursion amplitude is greater than the inter-atomic distance, multiphoton ionization effect will be reduced because of very frequent collisions in the solid, as we will show later.

Also notice that there is no observable difference on the breakdown threshold for the linearly and circularly polarized light for pulse width of $55 \mathrm{fs}$ and $100 \mathrm{fs}$. The breakdown threshold for $55 \mathrm{fs}$ pulses is $8.0 \mathrm{~J} / \mathrm{cm}^{2}$ and $12.0 \mathrm{~J} / \mathrm{cm}^{2}$ for $100 \mathrm{fs}$ pulses. These correspond to intensities of $1.45 \times 10^{14} \mathrm{~W} / \mathrm{cm}^{2}$ and $1.2 \times 10^{14} \mathrm{~W} / \mathrm{cm}^{2}$, respectively. This result is, again, in contradiction to the multiphoton ionization theory. Previous experiment by Lompré et al. showed that the ionization rate for linearly and circularly polarized light is quite different [15]. The linearly polarized light can ionize much more efficiently than the circularly polarized light when ionization is through fourth or higher order photon absorption. This

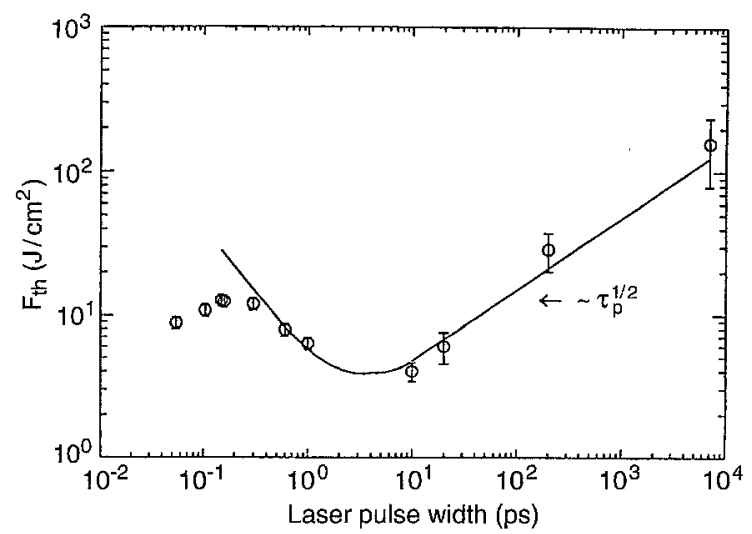

Fig. 1. The breakdown threshold as a function of pulse width. Solid line is based on the avalanche ionization theory. At pulse widths of $55 \mathrm{fs}$ and $100 \mathrm{fs}$, the difference of breakdown threshold between circularly and linearly polarized light is within experimental error was explained by Gontier and Trahin [16]. It is understandable that due to the conservation of angular momentum, the channels to the final states are much fewer for circularly polarized light. Therefore, linearly polarized light should have a much smaller breakdown threshold than that of circularly polarized light, if multiphoton ionization process is uninterrupted as it is in low density gases.

This contradiction can be explained if we realized that we used solid state material as our target, whereas gases were used by Lompré et al. and MPI is suppressed in solids when the electron excursion amplitude becomes bigger than the inter-atomic distance as our numerical calculation shows below.

In order to study the scattering effect on MPI, we numerically integrated the time-dependent Schrödinger equation. Scattering effect in MPI has been simulated by the Monte Carlo method. To our knowledge, collisional effect of a bound electron with neighboring atoms in the MPI process has not been addressed before.

Laser-atom interaction can be described by the timedependent Schrödinger equation

$i \hbar \frac{\partial \boldsymbol{\Psi}(\mathbf{r}, t)}{\partial t}=\left[\frac{\left(\mathbf{P}-\frac{e}{c} \mathbf{A}(\mathbf{r}, t)\right)^{2}}{2 m}+V(r)\right] \boldsymbol{\Psi}(\mathbf{r}, \mathbf{t})$,

where $V(r)$ is the coulomb potential, $\mathbf{P}$ is the electron momentum, and $\mathbf{A}(\mathbf{r}, t)$ is the vector potential of the laser field. Expanding (1), we have

$$
\begin{aligned}
i \hbar \frac{\partial \boldsymbol{\Psi}(\mathbf{r}, t)}{\partial t}= & \frac{P^{2}}{2 m} \boldsymbol{\Psi}(\mathbf{r}, t)+\frac{e}{2 m c}(\mathbf{A} \cdot \mathbf{P}+\mathbf{P} \cdot \mathbf{A}) \boldsymbol{\Psi}(\mathbf{r}, t) \\
& -\frac{e^{2} A^{2}}{2 m c^{2}} \boldsymbol{\Psi}(\mathbf{r}, t)+V(r) \Psi(\mathbf{r}, t) .
\end{aligned}
$$

In the dipole approximation, the laser field is spatially uniform, $\mathbf{A}(\mathbf{r}, t)=\mathbf{A}(\mathbf{R}, t)=\mathbf{A}(t)$, where $\mathbf{R}$ is the coordinate of the nucleus. Then

$\mathbf{P} \cdot \mathbf{A}=\mathbf{A} \cdot \mathbf{P}-\mathbf{i} \hbar \nabla \cdot \mathbf{A}=\mathbf{A} \cdot \mathbf{P}$.

Equation (1) becomes:

$$
\begin{aligned}
\mathrm{i} \hbar \frac{\partial \boldsymbol{\Psi}(\mathbf{r}, t)}{\partial t}= & \frac{P^{2}}{2 m} \boldsymbol{\Psi}(\mathbf{r}, t)+\frac{e}{m c}(\mathbf{A} \cdot \mathbf{P}) \boldsymbol{\Psi}(\mathbf{r}, t) \\
& -\frac{e^{2} A^{2}}{2 m c^{2}} \boldsymbol{\Psi}(\mathbf{r}, t)+V(r) \Psi(\mathbf{r}, t) \\
= & \frac{P^{2}}{2 m} \boldsymbol{\Psi}(\mathbf{r}, t)+\frac{e}{m c}(A \cdot P \cos \theta) \Psi(\mathbf{r}, t) \\
& -\frac{e^{2} A^{2}}{2 m c^{2}} \boldsymbol{\Psi}(\mathbf{r}, t)+V(r) \Psi(\mathbf{r}, t),
\end{aligned}
$$

where $\theta$ is the angle between $\mathbf{A}$ and $\mathbf{P}$.

First, we solved the time-dependent Schrödinger equation numerically without including collisions to test our simulation. We followed the approach used by Kulander [10]. The Alternating-Direction-Implicit (ADI) method [17] is used to solve (3). We integrated the Schrödinger equation for 20 periods. The laser field took five periods to 
"ramp up" and was held constant for the next fifteen cycles.

$E(t)= \begin{cases}\frac{1}{t_{0}} E_{\max } \sin \omega t, & t \leq t_{0}, \\ E_{\max } \sin \omega t & t>t_{0},\end{cases}$

where $E_{\max }$ is the maximum electric field, $\omega$ is the angular frequency of the laser light, $t_{0}=5 T$, and $T$ is the optical period. Since

$A=-\int E(t) \mathrm{d} t$,

we have

$A(t)= \begin{cases}E_{\max } \frac{t \cos \omega t}{\omega t_{0}}-E_{\max } \frac{\sin \omega t}{\omega^{2} t_{0}}, & t \leq t_{0}, \\ -E_{\max } \frac{\sin \omega t_{0}}{\omega^{2} t_{0}}+E_{\max } \frac{\cos \omega t}{\omega}, & t>t_{0} .\end{cases}$

A cylindrical symmetry is assumed and a two-dimensional grid in $\rho z$ space is chosen. The size of the integration grid is $25 \times 65$ (atomic unit). A short-range imaginary absorption potential was set at the outer boundary to absorb particles so that we do not have numerical reflections. The model atom is a hydrogen-like atom with an ionization potential of $9 \mathrm{eV}$ (which is the ionization potential of $\mathrm{SiO}_{2}$ ). The laser intensity in this calculation is $1.96 \times 10^{14} \mathrm{~W} / \mathrm{cm}^{2}$, and wavelength is $780 \mathrm{~nm}$. The result is shown in Fig. 2. We plotted the probability function $P(t)=\int 14 r^{2} d^{3} r$ against time. It is clear that $P(t)$ decreases as the laser field is turned on, which means that the atom is ionized. The ionization rate is determined through the exponential decay of the density of the probability function. We have compared the ionization rate of hydrogen obtained by us to that obtained by Kulander, and they agree very well. We are confident that our numerical code is working correctly.

When laser interacts with a solid, scattering (collision) inside the solids is very frequent [18]. There are a few scatterings during an optical cycle (on the order of 10 ).

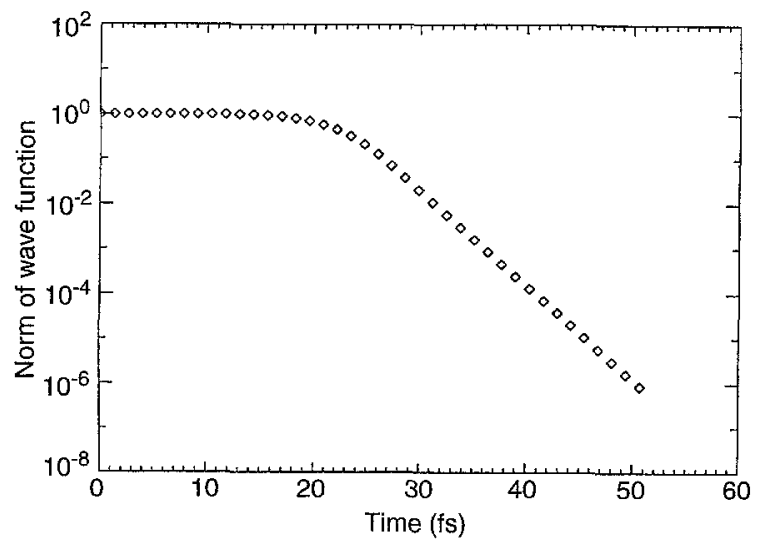

Fig. 2. The norm of the wave function decreases as the laser field's turned on. It indicates that the atom is ionized. The exponential decay of the norm gives a well defined ionization rate. In this case, the intensity of the laser is $1.91 \times 10^{14} \mathrm{~W} / \mathrm{cm}^{2}$, and the ionization frequency is $4.88 \times 10^{14} \mathrm{~s}^{-1}$
The periodic motion of electrons has been disturbed, and the electrons are dephased with respect to the driven field. We have used Monte Carlo simulation to study the scattering (collision) effect. The scattering potential is assumed to be similar to that in the screened Rutherford scattering. For a scattering potential

$U(r)=\frac{Z e^{2} \mathrm{e}^{(-\lambda r)}}{r}$

in which $Z$ is the atomic number and $\lambda$ is a variable quantifying the decay of the potential with distance, the scattering cross-section is given by

$\sigma(\theta)=\frac{e^{4} Z^{2}}{p^{2} v^{2}} \frac{1}{\left(1-\cos \theta+2 \eta_{\mathrm{s}}\right)^{2}}$

in which $p, v$ are the momentum and speed of the electron, respectively, and $\eta_{\mathrm{s}}$ is the screening parameter given by

$\eta_{\mathrm{s}}=\frac{1}{4} \frac{\lambda^{2}}{p^{2}}$

The probability distribution function is then

$p(\theta)=\frac{2 \eta_{\mathrm{s}}\left(1+\eta_{\mathrm{s}}\right)}{\left(1-\cos \theta+2 \eta_{\mathrm{s}}\right)^{2}}$.

Each scattering is treated as a distinct event giving rise to the angular deflection of the electron. We considered only elastic scattering and assumed that the electron does not lose its kinetic energy through this scattering because of the huge mass difference between the electron and the ions. After scattering, the momentum of the electron $\mathbf{P}$ will change its direction. As we can see from (3), only the term $\mathbf{A} \cdot \mathbf{P}$ will be affected. It is, therefore, equivalent to change the direction of the vector potential A instead of changing the direction of $\mathbf{P}$. The occurrence of collisions is random and is sampled by a random number generator. A second random number generator is used to sample the deflection angle according to the probability distribution function $p(\theta)$. We assume that collision happens instantaneously. The angle between the vector potential and momentum after collision is changed to a new value which remain constant until the next collision changes it. We have used 10 collisions per optical cycle in this calculation.

The result is shown in Fig. 3. It is clear that without collisions, the ionization rate is defined by observing the exponential decay of the probability function $P(t)$ (see Fig. 2). However, when collision is considered, the decay of the probability function $P(t)$ slows down, revealing the reduction of ionization. Due to the statistical behavior of the Monte Carlo method, the ionization rate is different for different scattering events, but all of them are less than that without collision. In Fig. 4, we show the averaged $|\Psi(r, t)|^{2}$ for 30 different scattering events as a function of time compared to that without collision. It shows that a substantial reduction of MPI occurs when collisions are present. In order to obtain a statistically accurate result, it is necessary to run many cases. Nonetheless, we believe that, in principle MPI is suppressed by collisions inside solids.

The reduction of MPI can be understood as follows: since an electron can only absorb photon(s) by exchanging 

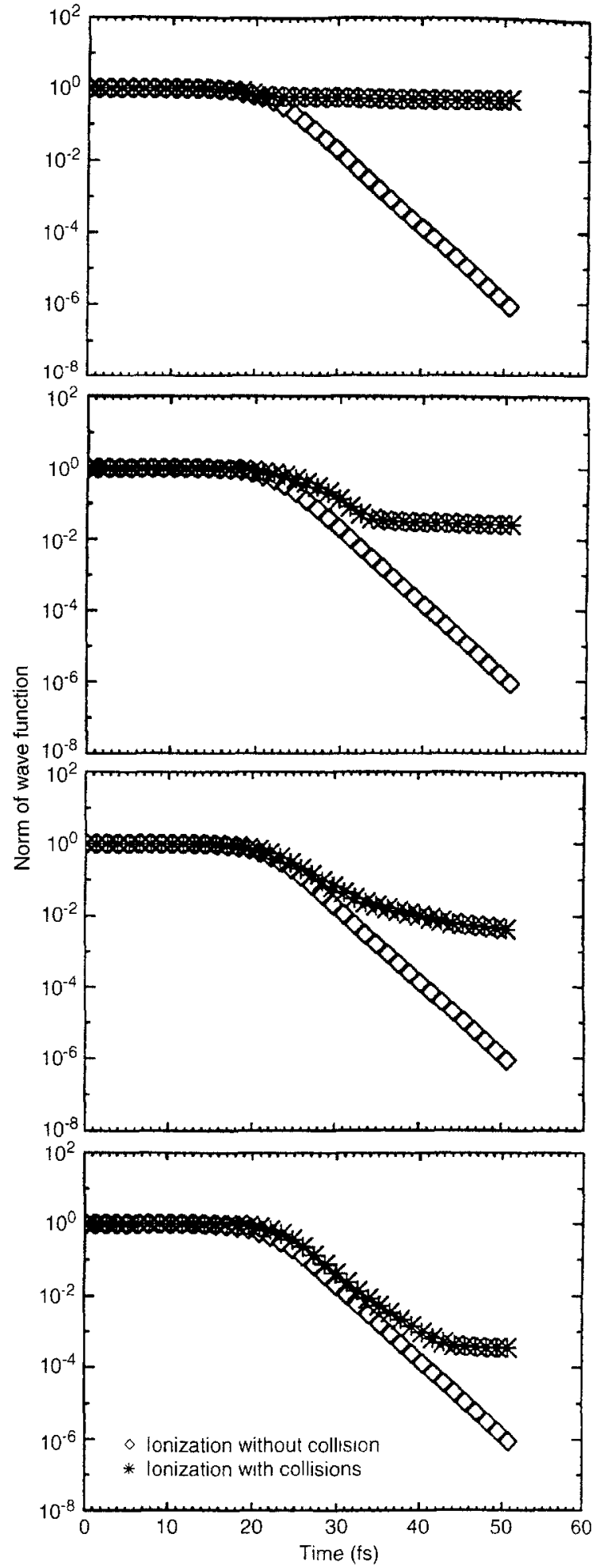

Fig. 3. Ionization with collisions. In these four different scattering cases, the ionization rate decreases due to the collisions inside solid

momentum with the nucleus, it has a bigger probability of being ionized when it is closer to its nucleus. In the absence of scattering, the electron moves around the nucleus in periodic orbits, and ionization probability goes high once every period. These ionized wave packets constructively interfere with each other to form a state of free

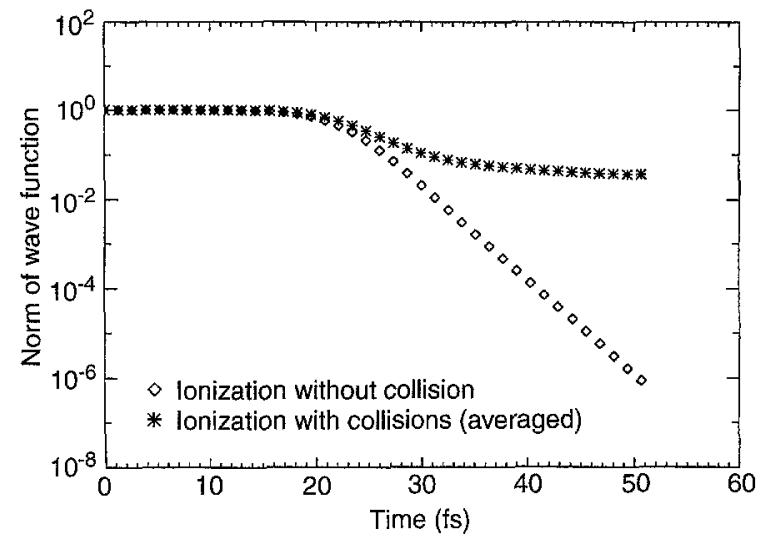

Fig. 4. Averaged ionization rate with collisions compared to that without collision. Substantial reduction due to collisions is readily observed

electron with kinetic energy of $n \hbar \omega-U_{I}-U_{\mathrm{p}}$, where $n$ is an integer. When collision happens, however, this periodic motion is disturbed and electrons have less chance to be close to the nuclei to absorb photons. When the ionized wave packets are out of phase with each other, they then lead to a destructive interference. Therefore the multiphoton ionization rate is reduced.

In conclusion, we first investigated the effect of collision to the multi-photon ionization process in a dielectric material through a laser-induced breakdown experiment. The high breakdown threshold for short pulses and the independence of the breakdown threshold on the polarization of the light can be explained by the suppression of multi-photon ionization. We have numerically integrated the time-dependent Schrödinger equation with scattering simulated by Monte Carlo method. A substantial reduction of the photoionization rate is observed due to the collisional effect. It suggests that the scattering effect becomes increasingly important when the laser and solid interact above the intensity level where the electron excursion amplitude is larger than the inter-atomic distance. It also reinforces the idea that dielectric breakdown is an avalanche ionization dominated process.

This work was supported by the National Science Foundation through the Center for Ultrafast Optical Science, contract number PHY8920108.

\section{References}

1. G.S. Voronov, N.B. Delone; JETP Lett, 1, (2), 42 (1965).

2. See papers in Multiphoton Ionization of Atoms, ed. by S.L. Chin and P. Lambropulos also in Atoms in Intense Laser Fields, ed. by M. Gavrila (Academic Press, New York, 1992) Academic Press, New York 1984).

3. G. Petite, J. Morrelec, D. Normand: J. Physique 40, 115 (1979)

4. P. Agostini, F. Fabre, G. Mainfray, G. Petite, N.K. Rahman: Phys. Rev. Lett. 42, 1127 (1979)

5. P. Kruit, J. Kimman, H.G. Muller, M.J. van de Wiel: Phys. Rev. A 28, 248 (1983).

6. R.R. Freeman, P.H. Bucksbaum, H. Milchberg, S. Darack, D. Schumacher, M.E. Geusic: Phys. Rev. Lett. 59, 1092 (1987)

7. L.V. Keldysh: Sov. Phys. JETP 20, 1307 (1965) 
8. F.H.M. Faisal: J. Phys. B: Atom. Molec. Phys, 6, L89 (1973)

9. H.R. Reiss: Phys. Rev. A 22, 1786 (1980)

10. K. Kulander: Phys. Rev. A 35, 445 (1987)

11. Q. Su, J. Eberly: Phys. Rev. A 43, 2474 (1991)

12. D. Du, X. Liu, G. Korn, J. Squier, G. Mourou: Appl. Phys. Lett. 64, $3071(1994)$

13. D. Du, J. Squier, S. Kane, G. Kane, G. Korn, G. Mourou, C. Bogusch, C. Cotton: Opt. Lett. (accepted for publication)
14. K.K. Thornber: J. Appl. Phys. 52, 279 (1981)

15. L.A. Lompé, G. Mainfray, C. Manus, J. Thebault: Phys. Rev. A 44, 1859 (1977)

16. Y. Gontier, M. Trahin: Phys. Rev. A 7, 2069 (1973)

17. S.E. Koonin, K.T.R. Davies, V. Maruhn-Rezwani, H. Feldmeier, S.J. Krieger, J.W. Negele: Phys. Rev. C 15, 1359 (1977)

18. M.V. Fischetti, D.J. DiMaria: Solid-State Electron. 31 (3/4) 629 (1988) 